\title{
Bemerkungen zur 4. Auflage
}

Zur Partie 24 gibt L. Stadtbäumer, Münster, an, daß nach $25 . \ldots$ Kg4: das Matt schon in vier Zügen erfolgt: 26. Dg6† Kf3 (Kh4 27. Dg5† und 28. Dg3 matt) 27. Dg3† Ke4 28. Tel† Kd4 29. De3 matt oder 28. . . Kf5 29. Dg5 matt.

Der gleiche Leser erkannte zwei analytische Irrtümer in Partie 57. Das beim 24. Zug vorgeschlagene 24. Dc2 Lf6 25. Sel ist ein grober Fehler wegen 25. ... Sel:! und Schwarz gewinnt: a) 26. Td7: Dg2: matt; b) 26. Dg6: Td2:; c) 26. Tel: Td2:. Das beim 25. Zug in Variante II in Klammern er. wähnte 25. ... Sd3 26. Td3: beantwortet Schwarz mit Dd3:! und Vorteil. Weiß müßte also etwa 26. Db6 ziehen, um sich günstig zu stellen.

Partie 8. Der in der Anmerkung zum 5. Zug von Schwarz in Klammern erwähnte Zug 9. ... Da5 gilt wegen 10. Tb1 Ld2: † 11. Dd2: Dd2† 12. Kd2: 0-0 13. Lb5 (Rubinstein-Schlechter, San Sebsstian 1912) als weniger gut. Anstelle von 6. . . Le6 empfiehlt J. I. Neistadt 6. . . cd 7. Sd4: Le7. Besser als 13. . . Td8 ist der aus einer Fernpartie 1947/1948 bekannte Zug 13. ... 0-0-0!, der nach Neistadt zu gleichem Spiel führt. Deswegen ist wohl 9. Se4: stärker als 9. Lb5†.

Partie 9. In der Analyse zum 17.Zug von Schwarz ist außer 21. ... Tac8. das sofort verliert, 21. . . Te6 zu er- wähnen. Weiß bleibt dann mit 22. Tc6: Dc6: 23. Dg4 Tg8 24. Dg8: $†$ im Vorteil.

Partie 10. Die Korrektheit des weiBen Opferspiels ist wegen 18. ... De5: fragwürdig, zum Beispiel 19. Dd8: Lf5:, oder 19. Dh5† Kf8.

Partie 13. In der Analyse zum 19. Zug ist 22. Lf7:t, das die Dame erobert, stärker als 22. Sh6:†.

Partie 15. Anmerkung zum 7. Zug. Der Beginn des zweiten Satzes muß lauten: „Nach e7-e6, gefolgt von Lf8-e 7 und $0-0$....".

Partie 17. Zum Schlußzug ist zu bemerken, daB 33. Ted8: (also das Schlagen mit dem anderen Turm) die Rettung 33. ... Del† ermöglicht.

Partie 20. Nach Pachman ist der Zug 4. Da4 wegen 4. . . Lf3: 5. ef e6 6. Sc3 S8e7 7. Le3 g6 fragwürdig. Anstelle von $9 . \ldots$ Tb8 führt $9 . \ldots 0-0$ ! 10. Tb7: e5! (11. Lg5 Dc8) zum Ausgleich.

Partie 21. Anmerkung zum 11. Zug von Schwarz. Die angedeutete Befreiungaaktion 12. Sld2 Se4: 13. Se4: d5 kostet wegen 14. Ld5: Dd5: 15. de einen Bauern. Schwarz müßte also im 12. Zug den Bauerntausch auf d4 vorausschicken.

Partie 29. Die heutige Theorie hält 4. Lf4 für aussichtsreicher.

Partie 62. Der 8. Zug lautet Dd1--

e2t. 
